(c) American Dairy Science Association, 2004.

\title{
Occurrence of Polycyclic Aromatic Hydrocarbons in Smoked Cheese
}

\author{
María D. Guillén and Patricia Sopelana \\ Tecnología de Alimentos, \\ Facultad de Farmacia, \\ Universidad del País Vasco, \\ Paseo de la Universidad, \\ n 7, 01006 Vitoria, Spain
}

\section{ABSTRACT}

The presence of polycyclic aromatic hydrocarbons (PAH) in smoked cheeses of different origin was studied. The samples were subjected to an initial extraction of fat and an alkaline treatment, extracted with cyclohexane, cleaned up by means of solid-phase extraction tubes, and analyzed by gas chromatography/mass spectrometry (GC/MS) operating in selective ion-monitoring mode (SIM). The results revealed the presence of numerous polycyclic aromatic hydrocarbons in the exterior zone of the samples, some of them with methyl groups. In all cases, the concentrations of compounds of low molecular weight were much higher than those of high molecular weight. Polycyclic aromatic hydrocarbons with varying degrees of carcinogenicity were identified, including benzo(a)pyrene in concentrations, which, although they did not exceed the limit established for this compound in the rind of ripened cheeses, do exceed the limit of 0.03 $\mu \mathrm{g} / \mathrm{kg}$ fixed for other foods smoked with smoke flavorings. Significant differences in the number and concentration of PAH in smoked cheese also were observed from rind to interior, the rind being the most contaminated zone. (Key words: polycyclic aromatic hydrocarbons, smoked cheese, gas chromatography-mass spectrometry)

Abbreviation key: GC/MS = gas chromatography/ mass spectrometry, PAH = polycyclic aromatic hydrocarbons, SIM = selective ion monitoring.

\section{INTRODUCTION}

Cheese is a foodstuff with a wide variety of types. Differences are due to the origin of raw material (ewe, buffalo, goat, cow, and so on) and to the manufacture, involving the type of rennet used, the use or not of mold, the ripening degree, and other factors. As in the manu-

Received June 10, 2003.

Accepted July 30, 2003.

Corresponding author: María Dolores Guillén; e-mail: knpgulod@ vf.ehu.es. facture of other commodities, smoking is an option in the process. In each European country, there are different well-known smoked cheese varieties much appreciated for their special organoleptic properties (Mcllveen and Vallely, 1996).

The smoking of cheese in European countries is carried out with smoke produced by different techniques, and some countries forbid the use of smoke flavorings to this end. Smoked foods are sometimes suspected of containing contaminants, such as polycyclic aromatic hydrocarbons (PAH), harmful to human health (Guillén et al., 1997). Taking into account the high solubility of PAH in lipids and the high proportion of fat in cheese, it might be thought that these contaminants are easily retained during the smoking process. Even so, the occurrence of PAH in smoked cheese has been little studied (Joe, Jr., et al., 1984; Riha et al., 1992; Bosset et al., 1998), even though this commodity is very often consumed. For these reasons, this paper investigates the possible presence of $\mathrm{PAH}$ in 7 commercial types of cheese smoked by traditional techniques, in order to get an idea of the PAH contamination in the cheeses that are commonly consumed. The study was carried out by extraction of these contaminants from the cheese matrix, isolation and separation, identification and quantification by means of gas chromatography/mass spectrometry (GC/MS) operating in selective ion monitoring (SIM) mode. The PAH that were the focus of the study were every $\mathrm{PAH}$, with alkyl groups or not, possibly present in cheese and able to be detected by the method employed.

\section{MATERIALS AND METHODS}

\section{Samples}

The samples are seven types of commercial smoked cheese, designated as A, B, C, D, E, F, and G. They were manufactured with milk from cows, sheep, goats, or a mixture of them, and acquired in local supermarkets. Some details of the cheese samples are shown in Table 1 . To study the level of contamination of these commodities, we took ground aliquots from the exterior zone of 
Table 1. Some characteristics of the cheese samples subject of study.

\begin{tabular}{llllllll}
\hline & $\mathrm{A}$ & $\mathrm{B}$ & $\mathrm{C}$ & $\mathrm{D}$ & $\mathrm{E}$ & $\mathrm{F}$ & $\mathrm{G}$ \\
\hline Milk origin & Cow & Sheep & Cow & Sheep & Goat & Cow and sheep Sheep \\
Milk thermal treatment & Pasteurized & Unpasteurized & Pasteurized & Unpasteurized & Unpasteurized & Pasteurized & Unpasteurized \\
Dry extract & $50 \%$ & $60 \%$ & $45 \%$ & $60 \%$ & $55 \%$ & $56 \%$ & $65 \%$ \\
Fat content & $45 \%$ & $45 \%$ & $45 \%$ & $45 \%$ & $43 \%$ & $47 \%$ & $50 \%$ \\
Type of cheese $^{2}$ & Semi-cured & Cured & Semi-cured & Cured & Fresh & Semi-cured & Cured \\
\hline
\end{tabular}

${ }^{1}$ Expressed as percentage relative to the dry extract.

${ }^{2}$ According to the ripening time.

each piece of cheese. This exterior zone corresponds to approximately a $1-\mathrm{cm}$ width without rind, and it represents a percentage relative to the total weight of the piece of $46.07,41.63,58.26,33.30,57.14$, and 58.60 in samples A, B, C, D, F, and G, respectively. In the case of sample $\mathrm{E}$, which is a type of fresh smoked cheese, a well-delimited rind did not exist, so rind and exterior were taken together, representing $37.10 \%$ of the total weight of the cheese piece taken.

\section{Reagents and Materials}

The solvents employed were cyclohexane and methanol, both HPLC grade (99.9+\%). Other reagents and materials used were potassium hydroxide, anhydrous sodium sulphate, sodium tungstate dihydrate, sodium chloride, and Supelclean LC-Si SPE (solid phase extraction) Tubes $3 \mathrm{~mL}(500 \mathrm{mg}$ ). All solvents, reagents, and materials mentioned are commercially available from Aldrich (Steinheim, Germany), Panreac (Barcelona, Spain), and Supelco (Bellefonte, PA).

\section{Standards}

The PAH standards used were a commercial mixture of PAH standards dissolved in a mixture of dichloromethane:benzene (75:25), containing naphthalene, acenaphthene, acenaphthylene, fluorene, phenanthrene, anthracene, fluoranthene, pyrene, benzo(c)phenanthrene, benz(a)anthracene, chrysene, 7,12-dimethylben$\mathrm{z}$ (a)anthracene, benzo(b)fluoranthene, benzo(j)fluoranthene, benzo(k)fluoranthene, benzo(a)pyrene, indene(1,2,3-cd)pyrene, dibenz(a,h)anthracene, benzo(ghi)perylene, dibenzo(a,l)pyrene, dibenzo(a,i)pyrene, and dibenzo(a,h)pyrene in concentrations of approximately $500 \mu \mathrm{g} / \mathrm{mL}$; commercial individual cyclohexane solutions of 1,7-dimethylnaphthalene, 1,4-dimethylnaphthalene, 1,5-dimethylnaphthalene, 1-methylphenanthrene, 2,3-dimethylanthracene, 9,10-dimethylphenanthrene, 2-methylfluoranthene, 1-methylfluoranthene, 11H-benzo(c)fluorene, 1-methylpyrene, 3methylchrysene, 2-methylchrysene, 5-methylchrysene, 4-methylchrysene, 6-methylchrysene, 1-methylchry- sene, dibenz(a,j)anthracene, benzo(b)chrysene, picene, anthanthrene, coronene, and dibenzo(a,e)pyrene, in concentrations of $10 \mu \mathrm{g} / \mathrm{mL}$ approximately; and a mixture of pure PAH dissolved in dichloromethane, containing 2,6-dimethylnaphthalene, 2,3-dimethylnaphthalene, $o$ terphenyl, 2-methylanthracene, 9-methylanthracene, 3,6-dimethylphenanthrene, $m$-terphenyl, $p$-terphenyl, 11H-benzo(a)fluorene, 11H-benzo(b)fluorene, benzo(e)pyrene, and perylene, in concentrations ranging from 100 to $247.5 \mu \mathrm{g} / \mathrm{mL}$. Naphthalene- $\mathrm{d}_{8}$, acenaphthene- $\mathrm{d}_{10}$, phenanthrene- $\mathrm{d}_{10}$, pyrene- $\mathrm{d}_{10}, \quad p$-terphenyl- $\mathrm{d}_{14}$, chrysene- $\mathrm{d}_{12}$, and perylene- $\mathrm{d}_{12}$ were used as internal standards. The purity of these standards ranged from 97 to $99.5 \%$.

All the above-mentioned standards were used in the identification of $\mathrm{PAH}$ and their quantification. All pure standards and solutions were obtained from Sigma, Supelco, and Symta (Madrid, Spain).

\section{Methods}

Extraction of fat from the samples. A mixture of deuterated internal standards was added to 20 to $25 \mathrm{~g}$ of ground cheese sample, which were mixed with $80 \mathrm{~mL}$ of cyclohexane and extracted for $25 \mathrm{~min}$ in an ultrasonic bath. Afterwards, the liquid was filtered through a common paper filter and the sample was reextracted twice with $50 \mathrm{~mL}$ of cyclohexane for $20 \mathrm{~min}$ and filtered each time. The total extract was concentrated to a small volume by means of a rotary evaporator and subjected to an alkaline treatment.

Alkaline treatment of the extracts. Approximately $11.2 \mathrm{~g}$ of potassium hydroxide dissolved in $100 \mathrm{~mL}$ of a mixture of methanol and distilled water $(9: 1, \mathrm{vol} / \mathrm{vol})$ and boiling chips were added to the previous extract, and the whole mixture was refluxed for $4 \mathrm{~h}$.

Extraction of PAH. After the alkaline treatment, the samples were diluted with $100 \mathrm{~mL}$ of a mixture of methanol/water (8:2, vol/vol) and extracted by liquidliquid partition in a separator funnel 3 times with 80, 50 , and $50 \mathrm{~mL}$ of cyclohexane, respectively. The total extract was washed 3 times with $100 \mathrm{~mL}$ of a mixture of methanol/water (8:2, vol/vol), $100 \mathrm{~mL}$ of water plus 5 
$\mathrm{g}$ of sodium chloride, and $100 \mathrm{~mL}$ of water, respectively. After the washings, $100 \mathrm{~mL}$ of an aqueous solution of $11 \mathrm{~g}$ of sodium tungstate dihydrate was added and left several hours. Finally, the cyclohexane extracts were filtered, dried over anhydrous sodium sulphate, and concentrated to $1 \mathrm{~mL}$ by using a rotary evaporator at first and a nitrogen stream at the end.

Clean-up procedure. The clean-up of the extracts was carried out by means of Supelclean LC-Si SPE Tubes $3 \mathrm{~mL}$ (500 mg), commercially available from Supelco. The cyclohexane extract was passed through one of these silica tubes, and $\mathrm{PAH}$ were eluted with $9 \mathrm{~mL}$ of cyclohexane; this eluate was concentrated to $1 \mathrm{~mL}$ and analyzed by GC/MS. Following the analysis, the eluate was passed through a second silica tube, and eluted with 1 or 2 $\mathrm{mL}$ of cyclohexane first (fraction 1) and 9 or $8 \mathrm{~mL}$ of cyclohexane afterwards (fraction 2), depending on the sample. The second fraction from the second tube was again concentrated to $1 \mathrm{~mL}$ and analyzed by GC/MS in order to identify and quantify some $\mathrm{PAH}$, which are difficult to identify in the eluate from the first tube due to the presence of interfering compounds. Fraction 1 was discarded, since this fraction contains mainly low molecular weight $\mathrm{PAH}$, which had already been correctly identified and quantified in the eluate from the first tube.

Identification and quantification by GC/MS technique. This was carried out by means of a HewlettPackard gas chromatograph model HP 6890 Series, equipped with a Mass Selective Detector 5973 and a Hewlett-Packard Vectra XM Series 4 computer. The column used was a fused-silica capillary column $(60 \mathrm{~m}$ long $\times 0.25 \mathrm{~mm}$ i.d. $\times 0.25-\mu \mathrm{m}$ film thickness), coated with a nonpolar stationary phase (HP-5MS, 5\% phenyl methyl siloxane). The operation conditions were the following: the oven temperature was set initially at $50^{\circ} \mathrm{C}(0.50 \mathrm{~min}$ hold), increased to $130^{\circ} \mathrm{C}$ at $8^{\circ} \mathrm{C} / \mathrm{min}$ and again increased to $290^{\circ} \mathrm{C}$ at a rate of $5^{\circ} \mathrm{C} / \mathrm{min}$ ( $50 \mathrm{~min}$ hold); the temperatures of the ion source and the quadrupole mass analyzer were kept at 230 and $150^{\circ} \mathrm{C}$, respectively. Helium, with a purity of $99.999 \%$, was used as a carrier gas at a constant flow of $1.0 \mathrm{~mL} / \mathrm{min}$; injector and transference line temperatures were held at 290 and $300^{\circ} \mathrm{C}$, respectively; pulsed splitless mode was used for injection with a pressure pulse of $30 \mathrm{psi}$, and $1 \mu \mathrm{L}$ of each sample was introduced in the gas chromatograph. The data acquisition mode employed was scan and SIM. Scan mode was used to determine the type of compounds present in the samples, whereas SIM was used to identify and quantify the PAH present.

Identification of the compounds was based on their retention times and on the relative abundances of the ions selected for their identification. Quantification in SIM mode is based on the measurement of the peak area correspondent to the most abundant ion of each compound, and was carried out by means of the deuterated internal standards previously mentioned. Thus, naphthalene- $d_{8}$ was used for quantification of naphthalene and its methylderivatives, phenanthrene- $\mathrm{d}_{10}$ for phenanthrene, anthracene and their methylderivatives, pyrene- $\mathrm{d}_{10}$ for fluoranthene and pyrene, $p$-terphenyl- $\mathrm{d}_{14}$ for $m$-terphenyl, $p$-terphenyl, benzofluorenes and methyl-fluoranthenes/pyrenes, chrysene- $\mathrm{d}_{12}$ for benz(a)anthracene, chrysene and their methylderivatives, and last, perylene- $\mathrm{d}_{12}$ for PAH with higher molecular weights. The response factors of each compound relative to the internal standard chosen for its quantification were calculated for each sample.

\section{RESULTS AND DISCUSSION}

In relation to the methodology employed for the determination of $\mathrm{PAH}$ in the samples of smoked cheese, it must be pointed out that several trials were performed before choosing the scheme presented above. Thus, previous tests led us to the following conclusions: a) The initial extraction of fat from the samples makes the subsequent stages easier and, in general, it does not involve major losses of the extracted compounds, obtaining recovery yields of the deuterated internal standards added that range from $74.77 \%$ for acenaphthene- $\mathrm{d}_{10}$ to $88.60 \%$ for perylene- $\mathrm{d}_{12}$, except for naphthalene- $\mathrm{d}_{8}(40.02 \%)$, which is often subjected to higher losses because of its volatility; b) the alkaline treatment of the extracted fat is necessary to remove principally the fatty acids present in the extracts, which both interfere greatly in the determination of $\mathrm{PAH}$ and damage the equipment. The effect of this treatment can be seen in Figure 1, which shows the chromatograms of 2 extracts of sample A: one with an alkaline treatment prior to extraction (1.a) and the other without (1.b). It must be noticed that the large peak in Figure 1.a (a), identified as 3.-beta-cholest-5-en3 -ol, is removed during the clean-up process, whereas the acids present in Figure 1.b (b) remain through the whole process; $c$ ) the addition of sodium tungstate dihydrate is really effective to obtain a clear extract due to the precipitation of lipoproteins (Simko et al., 1993).

The PAH identified in the samples studied and their concentrations, given in $\mu \mathrm{g} / \mathrm{kg}$ of the exterior part, are given in Table 2 . These results come from duplicate analyses of two aliquots per sample. Table 2 also shows the total PAH concentration of each sample, as well as the ratio between the concentrations of phenanthrene and pyrene and the ratio between the concentrations of pyrene and benzo(a)pyrene. It can be observed from this table that a high number (50) of $\mathrm{PAH}$ with a wide range of molecular weights have been identified. Thus, both compounds of low molecular weight (naphthalene, acenaphthene, fluorene) and of high molecular weight (ben- 


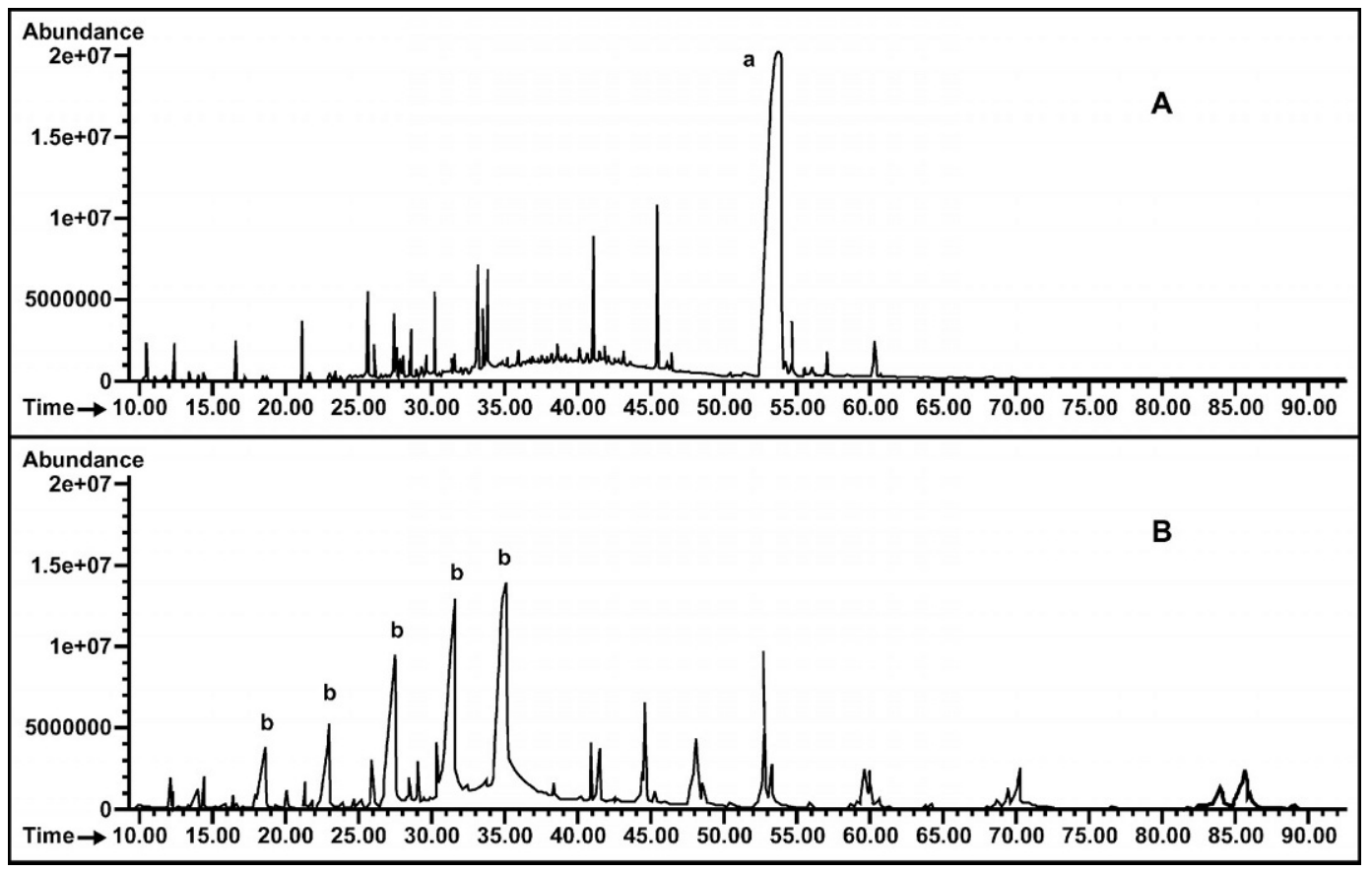

Figure 1. Total ion chromatograms of two extracts of sample A: one with a previous alkaline treatment (A) and the other, without (B). Peaks designated as (a) and (b) correspond to 3.beta-cholest-5-en-3-ol and acids, respectively.

z(a)anthracene, benzo(a)pyrene, benzo(ghi)perylene) are present, even though compounds of 2,3, and 4 aromatic rings are the most abundant. In fact, $\mathrm{PAH}$ with molecular weight higher than 228 , that is greater than ben$\mathrm{z}$ (a)anthracene, chrysene, or triphenylene, have only been found in samples A, B, and E basically. It was also noticed that more than half of the PAH identified have alkyl groups. In relation to the concentrations of the different PAH, it can be seen that they follow a similar pattern in all the samples studied; the highest concentrations correspond to the lightest PAH (naphthalene and their methyl derivatives, acenaphthylene, fluorene, phenanthrene) and, in general, as the molecular weight of the compounds increases, their concentrations decrease to very small $(0.04 \mu \mathrm{g}$ of benzo(ghi)perylene/kg of exterior), or even undetectable, values. With regard to alkylated $\mathrm{PAH}$, in general their individual concentrations are lower than those of their parent PAH, except for 2-methyl-naphthalene in sample $\mathrm{F}$ and some dimethylnaphthalenes in sample C. Nevertheless, it was noticed that, in the case of naphthalene, the total concentrations of both monomethyl and dimethyl derivatives are far higher than that of naphthalene in most of the samples, especially in sample C. In contrast, there are also samples in which the former concentrations are of the same order or lower than the latter, such as samples E and $\mathrm{G}$, respectively. In relation to other PAH having methyl groups, such as phenanthrene/anthracene or fluoran- thene/pyrene, it can be observed in Table 2 that the total concentrations of their methyl derivatives are, in general, of the same order or lower than that of their parent PAH, except for sample C, where the total of both methyl- and dimethyl-phenanthrenes are practically double the concentration of phenanthrene. An explanation for the high variability observed in the concentration of methyl derivatives in the samples of this study could be found in the nature of the wood used for the smoking process. Guillén et al. (2000b) studied several liquid smoke flavorings obtained from different types of wood and found that the flavoring coming from vine shoots contained high concentrations of naphthalene and their methyl derivatives in relation to that from other woods. It must be noticed that the distribution of the concentration of PAH in these samples matches well with the PAH pattern observed in smoke flavorings coming from the pyrolysis of wood (Guillén et al., 2000b).

Sample A has the highest number (49) and total concentration of PAH (1037.23 $\mu \mathrm{g} / \mathrm{kg}$ of exterior), whereas sample $G$ is the least contaminated in terms of total $\mathrm{PAH}$ concentration $(36.31 \mu \mathrm{g} / \mathrm{kg}$ of exterior). However, the samples with higher total concentrations of $\mathrm{PAH}$ do not always have higher numbers of PAH. Thus, sample C, with a total concentration of $367.53 \mu \mathrm{g} / \mathrm{kg}$ of exterior, has $28 \mathrm{PAH}$ and none of high molecular weight, whereas sample E, with $194.65 \mu \mathrm{g} / \mathrm{kg}$ of exterior of total PAH concentration, has $41 \mathrm{PAH}$, including high molecular 


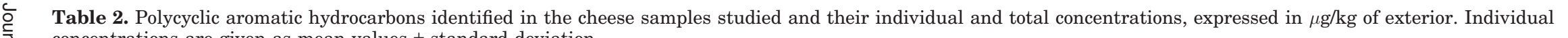
concentrations are given as mean values \pm standard deviation.

\begin{tabular}{|c|c|c|c|c|c|c|c|}
\hline & $\mathrm{A}$ & $\mathrm{B}$ & $\mathrm{C}$ & $\mathrm{D}$ & $\mathrm{E}$ & $\mathrm{F}$ & G \\
\hline Naphthalene & $175.74 \pm 12.11$ & $105.16 \pm 6.34$ & $31.41 \pm 2.23$ & $27.97 \pm 1.53$ & $40.14 \pm 4.16$ & $18.80 \pm 0.40$ & $9.32 \pm 0.35$ \\
\hline Methylnaphthalenes & 212.27 & 157.52 & 50.95 & 37.89 & 40.03 & 38.93 & 8.42 \\
\hline 2-Methylnaphthalene & $129.97 \pm 1.75$ & $97.59 \pm 7.80$ & $26.79 \pm 1.10$ & $24.33 \pm 0.11$ & $23.70 \pm 0.02$ & $25.73 \pm 2.15$ & $5.32 \pm 0.36$ \\
\hline 1-Methylnaphthalene & $82.30 \pm 0.25$ & $59.93 \pm 4.47$ & $24.16 \pm 1.64$ & $13.56 \pm 0.42$ & $16.33 \pm 0.30$ & $13.20 \pm 1.24$ & $3.10 \pm 0.13$ \\
\hline Dimethylnaphthalenes & 439.16 & 298.65 & 197.48 & 70.69 & 49.21 & 38.56 & 7.39 \\
\hline 2,6-Dimethylnaphthalene & $115.10 \pm 3.27$ & $88.62 \pm 6.84$ & $37.54 \pm 0.11$ & $21.22 \pm 0.84$ & $13.43 \pm 1.05$ & $13.94 \pm 0.98$ & $2.45 \pm 0.07$ \\
\hline 1,7-Dimethylnaphthalene & $91.63 \pm 6.47$ & $80.50 \pm 11.62$ & $31.41 \pm 2.30$ & $17.72 \pm 1.10$ & $10.73 \pm 0.78$ & $12.07 \pm 0.63$ & $2.30 \pm 0.04$ \\
\hline 1,6-Dimethylnaphthalene & $93.01 \pm 5.61$ & $58.85 \pm 4.16$ & $82.29 \pm 0.94$ & $14.43 \pm 0.73$ & $12.20 \pm 1.44$ & $10.05 \pm 0.26$ & $1.98 \pm 0.14$ \\
\hline 1,4-+2,3-Dimethylnaphthalene & $39.09 \pm 2.16$ & $39.78 \pm 2.83$ & $19.96^{6}$ & $9.17^{6}$ & $7.30 \pm 0.64$ & & \\
\hline 1,5-Dimethylnaphthalene & $21.01 \pm 1.91$ & $13.39 \pm 1.33$ & $14.91 \pm 0.18$ & $3.87 \pm 0.78$ & $2.40 \pm 0.09$ & $2.50 \pm 0.16$ & $0.66 \pm 0.33$ \\
\hline Dimethyl-ethyl-naphthalene & $79.32 \pm 5.29$ & $17.51 \pm 1.32$ & $11.37 \pm 0.42$ & $4.28 \pm 0.82$ & $3.15 \pm 1.18$ & & \\
\hline Acenaphthylene & $44.48 \pm 5.33$ & $31.54 \pm 4.08$ & $1.79 \pm 0.10$ & $4.28 \pm 0.23$ & $11.03 \pm 0.90$ & $1.31 \pm 0.02$ & $0.36 \pm 0.01$ \\
\hline Acenaphthene & $10.37 \pm 0.83$ & $7.27 \pm 1.22$ & $1.46 \pm 0.29$ & $3.30 \pm 0.08$ & $2.31 \pm 0.13$ & $0.85 \pm 0.06$ & $0.28 \pm 0.00$ \\
\hline Fluorene & $37.86 \pm 2.20$ & $17.52 \pm 1.23$ & $8.42 \pm 0.51$ & $8.05 \pm 0.14$ & $8.92 \pm 0.08$ & $2.03 \pm 0.04$ & $0.75 \pm 0.04$ \\
\hline Phenanthrene & $42.90 \pm 4.27$ & $16.03 \pm 1.83$ & $13.85 \pm 1.48$ & $6.65 \pm 0.03$ & $18.09 \pm 1.39$ & $3.76 \pm 0.27$ & $1.93 \pm 0.01$ \\
\hline Anthracene & $7.04 \pm 0.56$ & $4.05 \pm 0.95$ & $1.42 \pm 0.06$ & $1.23 \pm 0.11$ & $4.16 \pm 0.17$ & $0.44^{6}$ & $0.12 \pm 0.01$ \\
\hline Methyl-phenanthrenes-anthracenes & 36.30 & 10.80 & 26.85 & 7.46 & 11.56 & 3.82 & 2.79 \\
\hline 3-Methylphenanthrene & $7.20 \pm 0.49$ & $2.69 \pm 0.43$ & $5.54 \pm 0.76$ & $1.76 \pm 0.03$ & $1.91 \pm 0.11$ & $0.92^{6}$ & $0.66 \pm 0.06$ \\
\hline 2-Methylphenanthrene & $10.25 \pm 0.88$ & $3.24 \pm 0.67$ & $8.24 \pm 0.80$ & $2.44 \pm 0.04$ & $3.45 \pm 0.25$ & $1.20 \pm 0.06$ & $0.88 \pm 0.01$ \\
\hline 2-Methylanthracene & $5.85 \pm 0.33$ & $0.87 \pm 0.15$ & & & $1.76 \pm 0.02$ & & \\
\hline 9-Methylphenanthrene & $6.15 \pm 0.52$ & $2.24 \pm 0.38$ & $5.99 \pm 0.86$ & $1.74 \pm 0.00$ & $2.12 \pm 0.24$ & $0.95 \pm 0.04$ & $0.72 \pm 0.03$ \\
\hline 1-Methylphenanthrene & $6.85 \pm 0.35$ & $1.76 \pm 0.35$ & $7.08 \pm 0.70$ & $1.52 \pm 0.01$ & $2.32 \pm 0.10$ & $0.75 \pm 0.04$ & $0.53 \pm 0.00$ \\
\hline Dimethyl-phenanthrenes/-anthracenes & 13.75 & 4.93 & 30.16 & 5.04 & 1.05 & 3.58 & 2.16 \\
\hline Dimethylphenanthrene or isomer (1) & $1.16 \pm 0.71$ & $\ldots 5$ & & & $\ldots$ & & \\
\hline Dimethylphenanthrene or isomer (2) & $1.40 \pm 0.60$ & $0.70 \pm 0.08$ & $1.96 \pm 0.69$ & $0.55 \pm 0.04$ & $\cdots$ & $0.40 \pm 0.02$ & $0.27 \pm 0.04$ \\
\hline Dimethylphenanthrene or isomer (3) & $0.79 \pm 0.30$ & $0.48 \pm 0.08$ & $2.04 \pm 0.25$ & $0.52 \pm 0.02$ & $\ldots$ & $0.38 \pm 0.02$ & $0.19 \pm 0.02$ \\
\hline Dimethylphenanthrene or isomer (4) & $1.07 \pm 0.68$ & & & & $\ldots$ & & \\
\hline Dimethylphenanthrene or isomer (5) & $2.53 \pm 1.00$ & $1.84 \pm 0.40$ & $7.44 \pm 1.05$ & $1.97 \pm 0.13$ & $\cdots$ & $1.28 \pm 0.11$ & $0.81 \pm 0.00$ \\
\hline Dimethylphenanthrene or isomer (6) & $2.71 \pm 1.10$ & $1.07 \pm 0.21$ & $4.55 \pm 0.41$ & $1.12 \pm 0.02$ & $\ldots$ & $0.82 \pm 0.02$ & $0.53 \pm 0.06$ \\
\hline Dimethylphenanthrene or isomer (7) & $3.08 \pm 1.22$ & $0.84 \pm 0.16$ & $14.17 \pm 1.36$ & $0.88 \pm 0.06$ & $1.87 \pm 0.08$ & $0.70 \pm 0.09$ & $0.36 \pm 0.04$ \\
\hline Dimethylphenanthrene or isomer (10) & $1.01 \pm 0.62$ & & $\ldots$ & & & & \\
\hline$o$-Terphenyl & $2.24 \pm 0.32$ & $0.86 \pm 0.25$ & & $1.45 \pm 0.05$ & $0.36 \pm 0.05$ & $3.61 \pm 0.04$ & $0.76 \pm 0.13$ \\
\hline Fluoranthene & $5.22 \pm 0.11$ & $2.59 \pm 0.62$ & $1.09 \pm 0.01$ & $0.58 \pm 0.01$ & $2.50 \pm 0.06$ & $0.48 \pm 0.07$ & $0.44 \pm 0.01$ \\
\hline Pyrene & $4.83 \pm 0.29$ & $1.94 \pm 0.36$ & $1.52 \pm 0.07$ & $0.54 \pm 0.01$ & $1.81 \pm 0.12$ & $0.62 \pm 0.10$ & $0.79 \pm 0.02$ \\
\hline Methyl-fluoranthenes-pyrenes & 1.24 & 0.58 & 1.13 & $\ldots$ & 1.07 & 0.05 & 0.16 \\
\hline 2-Methylfluoranthene & $0.22 \pm 0.01$ & $0.07 \pm 0.01$ & $\ldots$ & $\ldots$ & $0.12 \pm 0.01$ & $\ldots$ & $\ldots$ \\
\hline Methylfluoranthene or isomer & $0.18 \pm 0.01$ & $0.06 \pm 0.01$ & $\ldots$ & $\ldots$ & $0.15 \pm 0.01$ & $\ldots$ & $\ldots$ \\
\hline Methylfluoranthene or isomer & $0.16 \pm 0.01$ & $0.07 \pm 0.01$ & $\ldots$ & $\ldots$ & $0.09 \pm 0.00$ & $\ldots$ & \\
\hline Methylfluoranthene or isomer & $0.21 \pm 0.02$ & $0.12 \pm 0.00$ & $0.26 \pm 0.06$ & $\ldots$ & $0.23 \pm 0.01$ & & $0.04 \pm 0.00$ \\
\hline Methylfluoranthene or isomer & $0.23 \pm 0.03$ & $0.14 \pm 0.00$ & $0.57 \pm 0.19$ & $\ldots$ & $0.24 \pm 0.01$ & $0.05^{6}$ & $0.08 \pm 0.01$ \\
\hline 1-Methylpyrene & $0.24 \pm 0.02$ & $0.12 \pm 0.01$ & $0.30 \pm 0.01$ & $\ldots$ & $0.24 \pm 0.04$ & $\ldots$ & $0.04 \pm 0.00$ \\
\hline $1-\mathrm{MFt}+11 \mathrm{H}-\mathrm{B}(\mathrm{a}) \mathrm{FL}^{1}$ & $0.94 \pm 0.03$ & $0.28 \pm 0.01$ & $\ldots$ & $\ldots$ & $0.68 \pm 0.04$ & $\ldots$ & $\ldots$ \\
\hline $11 \mathrm{H}-B e n z o(b)$ fluorene & $0.38 \pm 0.02$ & $0.08 \pm 0.01$ & $\ldots$ & $\ldots$ & $0.16 \pm 0.03$ & $\ldots$ & $\ldots$ \\
\hline $11 \mathrm{H}-\mathrm{Benzo}(\mathrm{c})$ fluorene & $0.18 \pm 0.01$ & $0.05 \pm 0.00$ & $\ldots$ & $\ldots$ & $0.12 \pm 0.01$ & $\ldots$ & $\ldots$ \\
\hline$m$-Terphenyl & $0.21 \pm 0.02$ & $0.14 \pm 0.04$ & $\ldots$ & $0.12 \pm 0.03$ & $0.14 \pm 0.01$ & $0.32 \pm 0.03$ & $0.33 \pm 0.01$ \\
\hline p-Terphenyl & $0.09 \pm 0.01$ & $0.05^{6}$ & $\cdots$ & $0.07 \pm 0.01$ & $0.08 \pm 0.01$ & $0.10 \pm 0.01$ & $0.11 \pm 0.03$ \\
\hline Benz(a)anthracene ${ }^{2}$ & $0.39 \pm 0.02$ & $1.17 \pm 0.44$ & $\cdots$ & & $0.28 \pm 0.01$ & $0.04 \pm 0.00$ & $0.05 \pm 0.03$ \\
\hline Chrysene + Triphenylene & $0.64 \pm 0.02$ & $1.23 \pm 0.38$ & $\cdots$ & $0.12 \pm 0.00$ & $0.46 \pm 0.02$ & $0.11 \pm 0.01$ & $0.11 \pm 0.01$ \\
\hline Benzo(b)fluoranthene $^{2}$ & $0.19 \pm 0.01$ & $0.54 \pm 0.12$ & & $\ldots$ & $0.09 \pm 0.00$ & & \\
\hline Benzo(j+k)fluoranthenes ${ }^{2}$ & $0.26 \pm 0.03$ & $1.57 \pm 0.24$ & $\cdots$ & $\cdots$ & $0.14 \pm 0.01$ & $\cdots$ & $\cdots$ \\
\hline Benzo(e)pyrene & $0.13 \pm 0.01$ & $\ldots$ & $\cdots$ & $\cdots$ & $0.13 \pm 0.01$ & $0.05^{6}$ & $\cdots$ \\
\hline
\end{tabular}

(Continued) 
weight compounds, such as benzofluoranthenes or benzo(ghi)perylene. In the same way, sample G, which is the least contaminated in terms of total PAH burden, has a similar or even higher number of $\mathrm{PAH}$ than other samples.

It must be noted that not only is the number and total concentration of PAH important, but so is the nature of the PAH implied in this contamination. Thus, some of the PAH identified in these samples (see Table 2), such as benzo(b,j,k)fluoranthenes or indene(1,2,3-cd)pyrene, have been considered by the IARC as "possibly carcinogenic to humans" (group 2B) (IARC, 1973; 1983) and others as "probably carcinogenic to humans" (group 2A); among these latter benz(a)anthracene, benzo(a)pyrene and dibenz(a,h)anthracene can be cited. Benzo(a)pyrene, which is one of the few PAH for which a legal limit exists in different types of food matrices (Guillén and Sopelana, 2003), has been detected in samples A, B, and E, in concentrations ranging from $0.08 \mu \mathrm{g} / \mathrm{kg}$ of exterior in $\mathrm{E}$ to $0.52 \mu \mathrm{g} / \mathrm{kg}$ of exterior in B. Several authors have found a relation between the concentration of benzo(a)pyrene and pyrene in charbroiled hamburgers (Greenberg et al., 1993) and in commercial liquid smoke flavorings (Guillén et al., 2000a). The existence of such a relation could be attributed to the fact that heavy PAH derive from lighter PAH by the addition of small units, such as acetylene to aryl radicals during pyrolysis (Frenklach et al., 1987). For this reason, samples with high concentrations of light PAH usually present higher concentrations of heavy PAH. Moreover, the existence of other relations between the concentrations of other PAH could be expected. Thus, in Table 2, as well as the ratio pyrene/ benzo(a)pyrene, the ratio between the concentrations of phenanthrene and pyrene is also shown. As was expected, the ratio phenanthrene/pyrene is of the same order in the samples of this study, except for sample G, where this value is lower. In relation to the ratio pyrene/ benzo(a)pyrene, it can be observed that it is very similar in samples A and E (24.15 and 22.63, respectively), whereas in sample B this value is much lower (3.73), implying a higher proportion of benzo(a)pyrene. The existence of certain relations between the concentrations of some PAH appears as a useful tool to estimate the level of high molecular weight PAH from the concentrations of lighter PAH, whose determination is usually easier, provided that no additional contamination occurs, apart from that rising from the pyrolysis process. It can also be noticed that, in sample B, the concentrations of heavy PAH are much higher than those expected. This could be attributed to the presence of residues of previous pyrolytic processes in the smoking chamber, since the pyrolysis of these residues would lead to the formation of additional heavy PAH and, consequently, to an increase in the $\mathrm{PAH}$ concentration of the samples. 
When trying to evaluate the contamination of the samples of this study, it must be taken into account that values in Table 2 correspond to the exterior of the piece of cheese, which represents a percentage of the total varying between $33.30 \%$ in sample D and $58.60 \%$ in G. It was assumed that the exterior could be the most contaminated edible zone because of its proximity to the rind, which is the part in direct contact with smoke during the smoking process. Nevertheless, it is necessary to study whether these values can be extrapolated to the rest of the cheese or if, on the contrary, there are differences in PAH concentrations through the whole sample. Therefore, to determine the degree of contamination of each part of the cheese, different parts of the same sample were studied: rind $(10.98 \%$ of the total weight of the cheese piece), exterior (46.07\%), middle (36.56\%), and interior $(6.39 \%)$. Table 3 shows the concentrations of PAH in different parts of sample A, selected because it was the most contaminated. This table reveals that the rind is the most contaminated part, with a very high number (68) and concentration of PAH (7273 $\mu \mathrm{g} /$ $\mathrm{kg}$ ). There are some PAH that have only been identified in this part of the sample, such as some methyl derivatives of phenanthrene/anthracene and benz(a)anthracene/chrysene, as well as other PAH of high molecular weight whose concentrations are low; perylene, benzo(b)chrysene, coronene, and dibenzopyrenes or isomers can be cited as examples. It must be pointed out that, among all the PAH identified in the rind, there are several compounds considered as "probably" or "possibly" carcinogenic to humans by the IARC (groups 2A and 2B, respectively), such as benz(a)anthracene, benzofluoranthenes, dibenz(a,h)anthracene, or dibenzo(a,e)pyrene, not identified in the exterior. Benzo(a)pyrene has also been identified, even though its concentration does not exceed the limit of $10 \mathrm{ppb}(\mu \mathrm{g} / \mathrm{kg})$ established for this compound in the rind of ripened cheeses in the Spanish legislation (B.O.E., 6-12-1985). One must highlight the presence in the rind of compounds that, although not classified as carcinogenic to humans by the IARC, have been considered carcinogenic or even very carcinogenic according to other classifications (Guillén and Sopelana, 2003). In spite of the high contamination of the rind, it must be taken into account that this part is commonly discarded when eating. In relation to the most commonly edible part of cheese, it can be seen from Table 3 that the main PAH burden appears in the exterior (1037.23 $\mu \mathrm{g} / \mathrm{kg}$ ), whereas the PAH concentrations in the middle and interior are relatively similar (153.70 and $88.96 \mu \mathrm{g} /$ $\mathrm{kg}$, respectively). As to the number of compounds identified, it can be said that there are some PAH in the exterior that have not been identified either in the middle or in the interior, such as 2-methylanthracene, some dimethyl-phenanthrenes, methyl derivatives of fluoran- thene and pyrene and, in general, $\mathrm{PAH}$ with molecular weight higher than 228 . In relation to middle and interior, it can be observed that the number of compounds is practically the same, and that fluoranthene and pyrene onwards, concentrations of PAH are very similar in both parts. Taking into account that high molecular weight $\mathrm{PAH}$, including benzo(a)pyrene, are only identified in the exterior zone, it could be considered that the concentration of these compounds in the whole sample corresponds exclusively to the exterior. Therefore, considering the proportion of exterior relative to the whole cheese in each sample (A: 46.07, B: 41.63, and E: $37.10 \%$ ), the resulting concentrations of benzo(a)pyrene in samples $\mathrm{A}, \mathrm{B}$, and $\mathrm{E}$ would be $0.09,0.22$, and $0.03 \mu \mathrm{g} / \mathrm{kg}$, respectively, none of which exceed the limit fixed for the rind. However, two of them are higher than the limit of 0.03 $\mu \mathrm{g} / \mathrm{kg}$ established in foods smoked with smoke flavorings (88/388/EEC,OJL184 of 15-7-1988).

There are very few studies on the occurrence of PAH in smoked cheese (Howard et al., 1966; Crosby et al., 1981; Joe, Jr., et al., 1984; Riha et al., 1992; Bosset et al., 1998), and some of them refer only to benzo(a)pyrene (Potthast, 1977; García et al., 1999). On the other hand, among the studies including a higher number of $\mathrm{PAH}$, some of them do not specify whether they refer to smoked or unsmoked cheese (Dennis et al., 1991; Lodovici et al., 1995). In relation to benzo(a)pyrene, which is the only compound studied by all the authors, it can be said that the levels found in the samples of this study are in the range of values found by others (nd-0.91 $\mu \mathrm{g} / \mathrm{kg}$ ), except for Bosset et al. (1998), who found concentrations of 4.1 and $4.2 \mu \mathrm{g} / \mathrm{kg}$ in some of the smoked cheeses studied. It must be mentioned that there is no clear pattern in the concentrations of $\mathrm{PAH}$ found by other authors. It is observed that phenanthrene, anthracene, ben$z$ (a)anthracene, fluoranthene, and pyrene are identified in most of the samples, and the most abundant PAH are, in general, phenanthrene, fluoranthene, and anthracene. However, the lightest PAH, such as naphthalene, which are the most abundant in the samples of this study, have been found in a lower number of samples. It can also be noted that benz(a)anthracene, which cannot be identified in two of the samples of this study (C and D), is present in all the unsmoked samples of cheese studied by Bosset et al. (1998). On the other hand, the presence of alkylated $\mathrm{PAH}$ is not reported by any authors. Some of the differences found among the results of different authors could be attributed to the contamination source of the cheese samples or to the chromatographic method and detection system used. However, in general, the concentrations of PAH in the samples of this study are in the range of the values found by other authors and, in some cases, are lower. 
Table 3. Polycyclic aromatic hydrocarbons identified in different parts of sample A and their individual and total concentrations, expressed in $\mu \mathrm{g} / \mathrm{kg}$. Individual concentrations are given as mean values \pm standard deviation.

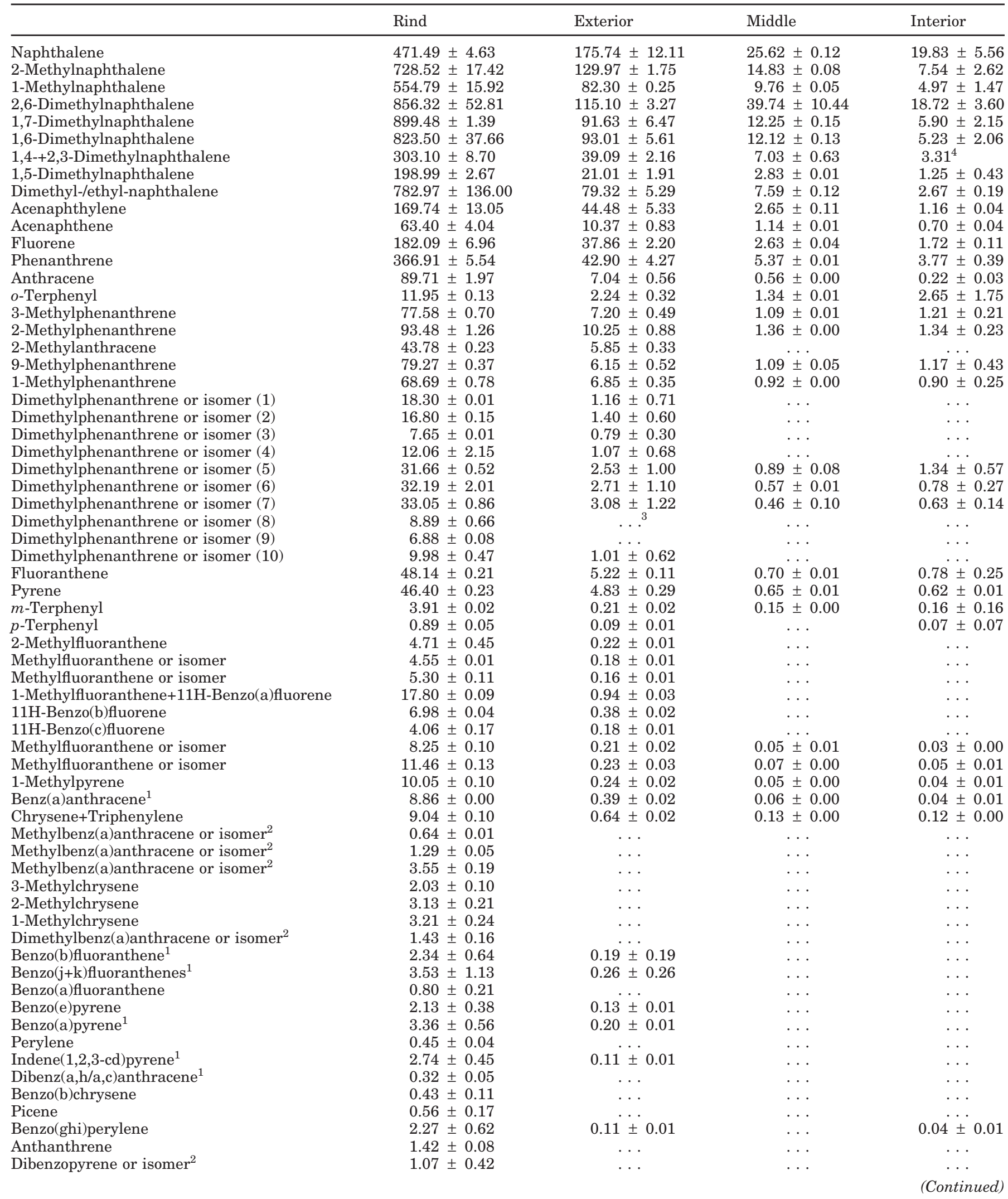


Table 3 Continued. Polycyclic aromatic hydrocarbons identified in different parts of sample A and their individual and total concentrations, expressed in $\mu \mathrm{g} / \mathrm{kg}$. Individual concentrations are given as mean values \pm standard deviation.

\begin{tabular}{|c|c|c|c|c|}
\hline & Rind & Exterior & Middle & Interior \\
\hline Coronene & $1.66 \pm 0.76$ & $\ldots$ & $\ldots$ & $\ldots$ \\
\hline $\operatorname{Dibenzo}_{(\mathrm{a}, \mathrm{e}) \text { pyrene }^{1}}$ & $0.36 \pm 0.11$ & $\ldots$ & $\ldots$ & $\ldots$ \\
\hline Dibenzopyrene or isomer $^{2}$ & $0.66 \pm 0.21$ & & & \\
\hline
\end{tabular}

${ }^{1}$ Compound with a certain degree of carcinogenicity, according to the International Agency for Research on Cancer (IARC, 1973; 1983).

${ }^{2}$ Tentative identification.

${ }^{3}$ Not identified.

${ }^{4}$ Identified only in one of the aliquots.

To sum up, it can be concluded that different PAH have been identified in the exterior of all the samples of this study, in variable concentrations. Given that smoking provides organoleptic properties that are much appreciated by consumers of cheese, great attention must be paid to avoid the incorporation of PAH to this foodstuff. For this purpose, the process must be carefully controlled in terms of wood pyrolysis temperature, and the smoking chamber must be kept clean in order to eliminate residues from previous pyrolysis, which can result in an additional contamination of the product. It is recommended that the rind be removed before consumption as a measure to avoid the ingestion of high levels of PAH.

\section{ACKNOWLEDGMENTS}

The authors gratefully acknowledge the financial support of the MCYT (AGL2000-1696), of the MAPA (CAL00-054-C3-2), and of the University of the Basque Country (9/UPV 00101.125.13667/2001).

\section{REFERENCES}

Bosset, J. O., U. Bütikofer, O. Dafflon, H. Koch, L. Scheurer-Simonet, and R. Sieber. 1998. Teneur en hydrocarbures aromatiques polycycliques de fromages avec et sans flaveur de fumée. Sci. Aliments 18:347-359.

Council Directive of 22 June 1988 on the approximation of the laws of the Member States relating to flavorings for use in foodstuffs and to source materials for their production (88/388/EEC, OJL184 of 15.07.1988).

Crosby, N. T., D. C. Hunt, L. A. Philp, and I. Patel. 1981. Determination of polynuclear aromatic hydrocarbons in food, water and smoke using high-performance liquid chromatography. Analyst 106:135-145.

Dennis, M. J., R. C. Massey, G. Cripps, I. Venn, N. Howarth, and G. Lee. 1991. Factors affecting the polycyclic aromatic hydrocarbon content of cereals, fats and other food products. Food Addit. Contam. 8:517-530.

Frenklach, M., and J. Warnatz. 1987. Detailed modelling of PAH profiles in a sooting low-pressure acetylene flame. Comb. Sci. Technol. $51: 265-283$.
García Falcón, M. S., S. Gonzlez Amigo, M. A. Lage Yusty, and J. Simal Lozano. 1999. Determination of benzo(a)pyrene in some Spanish commercial smoked products by HPLC-FL. Food Addit. Contam. 16:9-14.

Greenberg, A., C.-H. Hsu, N. Rothman, and P. T. Strickland. 1993. PAH profiles of charbroiled hamburgers: Pyrene/b(a)p ratios and presence of reactive PAH. Poycyclic Aromat. Compd. 3:101-110.

Guillén, M. D., P. Sopelana, and M. A. Partearroyo. 1997. Food as a source of polycyclic aromatic carcinogens. Rev. Environ. Health 12:133-146.

Guillén, M. D., P. Sopelana, and M. A. Partearroyo. 2000a. Determination of polycyclic aromatic hydrocarbons in commercial liquid smoke flavorings of different compositions by gas chromatographymass spectrometry. J. Agric. Food Chem. 48:126-131.

Guillén, M. D., P. Sopelana, and M. A. Partearroyo. 2000b. Polycyclic aromatic hydrocarbons in liquid smoke flavorings obtained from different types of wood. Effect of storage in polyethylene flasks on their concentrations. J. Agric. Food Chem. 48:5083-5087.

Guillén, M. D., and P. Sopelana. 2003. Polycyclic aromatic hydrocarbons in diverse foods. Pages 175-198 in Food Safety: Contaminants and Toxins. J. P. F. D'Mello, ed. CABI Publishing, Oxon, UK.

Howard, J. W., R. T. Teague, Jr., R. H. White, and B. E. Fry, Jr. 1966. Extraction and estimation of polycyclic aromatic hydrocarbons in smoked foods. I. General method. J. AOAC 49:595-611.

IARC. 1973. Certain polycyclic aromatic hydrocarbons and heterocyclic compounds. IARC Monographs on the Evaluation of the Carcinogenic Risk of Chemicals to Humans. Vol. 3. IARC, Lyon, France.

IARC. 1983. Polynuclear aromatic compounds, part 1: Chemical, environmental and experimental data. IARC Monographs on the Evaluation of the Carcinogenic Risk of Chemicals to Humans. Vol. 32. IARC, Lyon, France.

Joe, F. L., Jr., J. Salemme, and T. Fazio. 1984. Liquid chromatographic determination of trace residues of polynuclear aromatic hydrocarbons in smoked foods. J. AOAC 67:1076-1082.

Lodovici, M., P. Dolara, C. Casalini, S. Ciappellano, and G. Testolin. 1995. Polycyclic aromatic hydrocarbon contamination in the Italian diet. Food Addit. Contam. 12:703-713.

Mcllveen, H., and C. Vallely. 1996. The development and acceptability of a smoked processed cheese. Br. Food J. 98:17-23.

Orden de 29 de noviembre de 1985 por la que se aprueban las normas de calidad para quesos y quesos fundidos destinados al mercado interior (B.O.E. 6-12-1985).

Potthast, K. 1977. Polycyclic aromatic hydrocarbons in smoked meat products. An application of a new method. Acta Aliment. Pol. 3(27):195-201.

Riha, W. E., W. L. Wendorff, and S. Rank. 1992. Benzo(a)pyrene content of smoked and smoked-flavored cheese products sold in Wisconsin. J. Food Prot. 55:636-638.

Simko, P., S. Gergely, J. Karovicova, M. Drdak, and J. Knezo. 1993. Influence of cooking on benzo(a)pyrene content in smoked sausages. Meat Sci. 34:301-309. 